\title{
PROPUESTA DE METODOLOGÍA PARA LA INSERCIÓN DEL ESPÍRITU EMPRENDEDOR EN LA PLANEACIÓN DIDÁCTICA
}

\section{PROPOSED METHODOLOGY FOR THE INSERTION OF THE ENTREPRENEURIAL SPIRIT IN DIDACTIC PLANNING}

Olga del Socoro Rodríguez Lumbí ${ }^{1}$ Reynaldo Antonio Castaño Umaña ${ }^{2}$

(Recibido/received: 5-Febrero-2020; aceptado/accepted: 30-abril-2020)

RESUMEN: En Nicaragua, se han realizado valiosos esfuerzos por parte del estado, empresa privada y el sistema de educación, para fomentar y desarrollar el emprendimiento en el campo de la educación, tal es el caso del programa Nacional Educativo "Aprender, Emprender, Prosperar" (AEP), que integra los tres subsistemas educativos. Pese a esos esfuerzos se debe continuar profundizando en la implementación de estrategias que permitan la creación de un perfil educativo emprendedor en las diferentes casas de estudio. En ese sentido, es notoria la necesidad de una metodología que permita insertar el emprendimiento en los diferentes planes de estudio de las carreras universitarias, no necesariamente creando nuevas asignaturas, sino diseñando estrategias de aprendizaje que permitan el desarrollo y potencialización del fomento de la cultura emprendedora en los estudiantes. Con base en los referentes consultados, a través de esta investigación se propuso una metodología de trabajo, que permitió la identificación de estrategias de aprendizajes que incluyeron la potencialización de las características emprendedoras, considerando las unidades temáticas y los objetivos actitudinales que se llevan a cabo durante el desarrollo de las actividades académicas, describiendo la secuencia lógica a para el pertinente diseño de las estrategias, con la definición de los recursos didácticos necesarios para su implementación, así como la definición de los criterios a considerar por el docente para comprobar el desarrollo del espíritu emprendedor.

PALABRAS CLAVE: Emprendimiento, planificación didáctica, actividades de aprendizaje.

\footnotetext{
${ }^{1}$ Dirección de Desarrollo Educativo. Universidad Nacional de Ingeniería. Managua, Nicaragua. Correo electrónico : olglumbi@gmail.com

${ }^{2}$ Facultad de Ciencias y Sistemas. Universidad Nacional de Ingeniería. Managua, Nicaragua. Correo electrónico: reynaldo.castano@fcys.uni.edu.ni
} 
ABSTRACT: In Nicaragua, valuable efforts have been made by the state, private companies and the education system to promote and develop entrepreneurship in the field of education, such is the case of the National Educational program "Learn, Undertake, prosper" which integrates the three educational subsystems. Despite these efforts, the implementation of strategies that allow the creation of an entrepreneurial educational profile in the different study houses must continue. In this sense, the need for a methodology that allows inserting entrepreneurship in the different study plans of university degrees is notorious, not necessarily creating new subjects, but designing learning strategies that allow the development and empowerment of promoting entrepreneurial culture in the students. Based on the references consulted, through this research a work methodology was proposed, which allowed the identification of learning strategies that included the empowerment of entrepreneurial characteristics, considering the thematic units and the attitudinal objectives that are carried out during the development of the academic activities, describing the logical sequence a for the pertinent design of the strategies, with the definition of the didactic resources necessary for its implementation, as well as the definition of the criteria to be considered by the teacher to check the development of the entrepreneurial spirit.

KEYWORDS: Entrepreneurship, didactic planning, learning activities.

\section{INTRODUCCIÓN}

El emprendimiento no solo se refiere a la creación de nuevas empresas y/o negocios. Hoy en día, es entendida como una competencia que engloba un conjunto de habilidades y destrezas como son: la creatividad, el liderazgo, el trabajo en equipo, la innovación, la toma de decisiones; todas ellas demandadas en el ámbito personal, social y profesional.

Las instituciones educativas universitarias, facilitan la promoción de esta competencia a través de las asignaturas que conforman sus planes de estudios. No obstante, se encontró la siguiente limitante, ¿qué metodología se debería emplear para desarrollar está competencia a través de las asignaturas?, y ¿qué competencias, puede el docente, fomentar a través del desarrollo de su asignatura? El objetivo de este artículo fue responder a estas interrogantes realizando una revisión bibliográfica de los últimos artículos publicados, haciendo un recorrido por el término emprendimiento, para a continuación, señalar las habilidades y destrezas que engloba esta competencia, y el objetivo de la educación emprendedora.

De igual manera, se propuso determinar la valoración de los estudiantes, al desarrollar estrategias de aprendizaje que facilitaran el desarrollo de sus características emprendedoras, mediante la aplicación de la metodología propuesta a dos grupos de estudio de diferentes carreras y asignaturas, para validar la efectividad de la misma. Una vez comprobada la metodología, se procedió a capacitar a un grupo de docentes para que adoptaran la metodología en su planeación didáctica y fomenten el espíritu emprendedor en sus estudiantes. 


\section{METODOLOGÍA}

La metodología empleada para el desarrollo de esta investigación, constó de seis fases, las cuales se muestran en la figura 1.

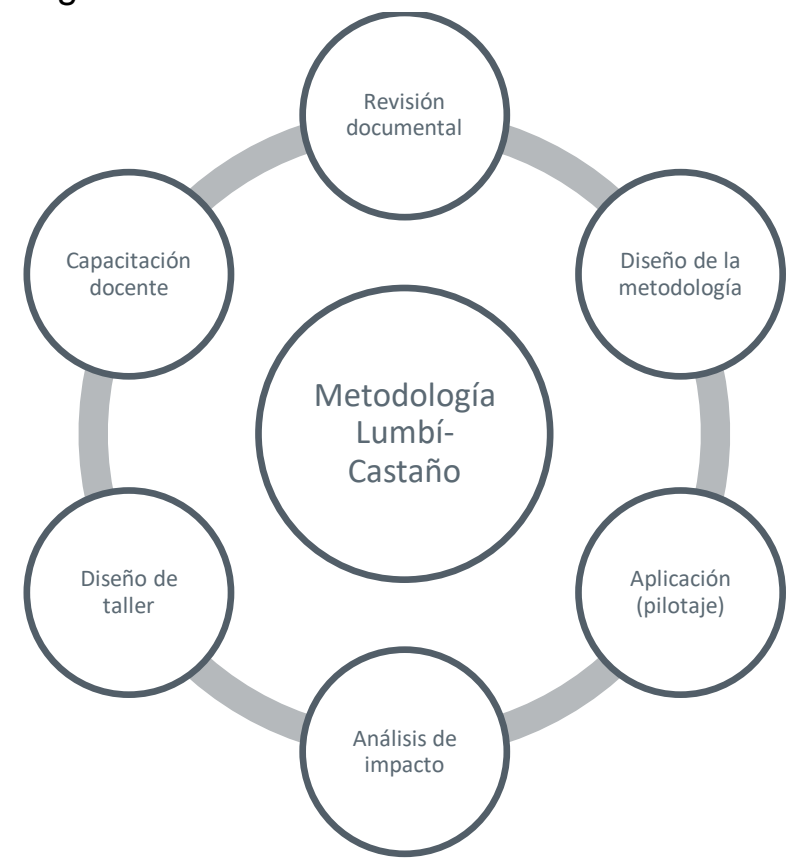

Figura 1. Metodología de trabajo.

Revisión documental: En esta fase se analizaron los precedentes documentales internacionales, acerca del emprendimiento y uso de estrategias de aprendizajes, analizando el estado del arte sobre el tema.

Diseño de la metodología: En esta fase, se procedió a analizar los elementos de los programas de asignaturas, planes calendario, estrategias de aprendizajes, componentes formativos, objetivos actitudinales, mecanismos de evaluación y su forma de integración para facilitar el desarrollo de las características emprendedoras.

Aplicación (pilotaje): La metodología propuesta, se aplicó en los planes didácticos de dos asignaturas, Química general, impartida en la carrera Ingeniería en electrónica de la Facultad de Electrotecnia y Computación con 27 estudiantes, y en Sistemas operativos, impartida en la carrera Ingeniería de Sistemas de la Facultad de Ciencias y Sistemas con 30 estudiantes, se procedió a ejecutar las estrategias de aprendizaje durante el primer semestre académico del año 2019.

Análisis del impacto: Una vez aplicada la metodología y ejecutadas las estrategias de aprendizaje, se procedió a determinar su impacto en el desarrollo de las características que definen el espíritu emprendedor, mediante la obtención de la apreciación de los estudiantes participantes en la fase anterior. Para el caso particular de los estudiantes que llevaron la asignatura de química general, la apreciación se obtuvo de forma verbal durante un 
conversatorio realizado al finalizar la ejecución de la estrategia de aprendizaje, mientras que a los estudiantes que llevaron la asignatura sistemas operativos, se aplicó una encuesta corta, a todo el grupo, consistente en cinco preguntas cerradas, mediante las cuales se obtuvo la apreciación y valoración del impacto.

Diseño del taller: Una vez que se validó la efectividad de la metodología, mediante la apreciación de los estudiantes, se procedió a realizar el diseño metodológico del taller a ser impartido a docentes de la universidad, con la finalidad de promover su utilización y la inserción del espíritu emprendedor en la planeación didáctica.

Capacitación docente: Una vez diseñado el taller y en coordinación con la Dirección de extensión de la UNI, se procedió a capacitar a un total de 40 docentes pertenecientes a las facultades: Ciencias y Sistemas, Arquitectura y Tecnología de la construcción; así como a 3 coordinadores de extensión, para un total de 43 docentes capacitados en la metodología.

\section{RESULTADOS Y DISCUSIÓN}

En un mundo de cambio constante y, continua evolución, es necesario desarrollar una serie de capacidades, y actitudes que permitan enfrentarse a las exigencias que demanda la sociedad. Para ello, se debe poner el foco en el aprendizaje a lo largo de la vida y la adquisición de competencias emprendedoras, especialmente en el desarrollo de los programas de educación universitaria. Diversas organizaciones internacionales hacen una llamada a las instituciones de Educación Superior a asumir la educación emprendedora para responder y anticiparse a las necesidades sociales.

A pesar del auge del emprendimiento en los últimos años, distintas investigaciones han confirmado que hay poca coherencia en el enfoque, sin existir un marco común sobre el concepto de emprender, ni un método referente en relación con la forma de enseñar o educar (Dacin et al. 2011; Fayolle y Gailly, 2008; Osorio y Pereira, 2011; Pfeilstetter, 2011; Sánchez, 2013). A esta necesidad detectada, se añade el hecho de que cada vez existe más interés por consensuar los conceptos claves que caractericen la formación y docencia en emprendimiento. Como señalan Ferrer et al. (2014, p14) "podemos encontrar puntos en común y un marco compartido cada vez más cercano".

Ante este desafío, se considera que las competencias pueden aprenderse y mejorarse (Sánchez, 2013; Taatila, 2010), y se sugiere que la formación debe ser formulada en términos de competencias (Miller, Wesley y Williams, 2012), incluyendo valores éticos, sociales y emocionales. Por ello, en el marco de la educación en emprendimiento, se ve fundamental el fomento de competencias que promuevan la creatividad, la imaginación, el pensamiento crítico, la colaboración, la proactividad y el trabajo en equipo. De esta manera, se promociona, a su vez, un fuerte sentido de autoestima y confianza (Koiranen y Ruohotie, 2001; Korsgaard, 2011; Pache y Chowdhury, 2012; Raposo y do Paço, 2011; Sánchez, 2013; Villanueva, 2013). Algunos autores ya reclaman que esta educación emprendedora debe ser compartida a lo largo de toda 
la institución universitaria, sin distinguirlo como propio únicamente de alguna escuela o facultad en particular. Además, apuntan que al menos se necesitan dos cambios para cumplir los objetivos de la educación emprendedora: se deben cambiar los planes de estudio y desarrollar métodos de enseñanza-aprendizaje distintos (Jones, Matlay y Maritz, 2012; Ruskovaara y Pihkala, 2013).

Tomando en cuenta lo antes mencionado, para el fortalecimiento de la formación académica en emprendimiento, es necesario que el personal docente ponga en práctica, metodologías, enfoques y estrategias didácticas que faciliten la formación de estudiantes proactivos, y permitan abordar en los procesos de aprendizaje; enfoques diferentes a los tradicionales. Como lo afirman Merino y Vargas (2011), "en todo plan formativo para el emprendimiento se debe considerar la interacción tanto de niveles individuales o psicológicos como institucionales y del entorno, pues todos influyen en la decisión de emprender".

Alcaraz R. (2015), Considera que "ser emprendedor es una forma de vida, basada en una lucha constante por transformar nuestros sueños en realidad". Los emprendedores se identifican por su habilidad para ser creativo y llevar a la realidad sus ideas, en otras palabras, abandonan por completo la costumbre y crean distintas maneras de potenciar lo que existe.

Para Aquije (2012), Emprender es una actitud y una aptitud. Emprender es una acción. Emprender es un verbo. Emprender es construir ideas y sueños; es planificar el pensamiento y activarlo hacia objetivos claros en forma creativa y proactiva.

En ese sentido, la Universidad Nacional de Ingeniería (UNI), asumió el compromiso de promover el espíritu emprendedor de los estudiantes, en las actividades académicas desarrolladas en el proceso de formación de ingenieros y arquitectos, es por ello que, en el proceso de actualización de los programas de asignaturas realizado en el año 2014, se incluye como uno de los cinco componentes formativos a desarrollar. Por tanto, se propone una metodología, que permite la inserción del espíritu emprendedor en la planeación docente a través del diseño de actividades de aprendizaje creativas que permitan el desarrollo, fortalecimiento y evaluación de las características emprendedoras.

Actualmente la literatura sobre emprendimiento, afirma que el emprendedor es depositario del denominado "espíritu emprendedor", entendido como un conjunto total de atributos, habilidades y actitudes que diferenciarán al emprendedor del resto de individuos con tareas afines. Las actitudes no son conductas sino predisposiciones adquiridas para actuar selectivamente, conducirse de determinada manera. Toda actitud posee aspectos cognitivos (creencias y opiniones hacia el objeto o situación); un aspecto afectivo (componentes afectivos de las creencias y opiniones, afectos que generan atracción o rechazo) y un aspecto normativo (como "debe" el sujeto comportarse ante determinada situación u objeto). Por lo anterior, se entenderá que el comportamiento o acción emprendedora lleva en gran parte como motor a nuestras actitudes. 
Luego de un análisis exhaustivo referencial sobre la temática del emprendimiento y su incorporación en la planeación didáctica, se llega a la conclusión que aún no existe una metodología u procedimientos de trabajo, que le permitan al personal docente incluir en el proceso de planeación didáctica, mecanismos de identificación, ejecución y evaluación de dicho componente formativo. Ante esta necesidad imperante, se propone la metodología LumbíCastaño, definida de la siguiente forma (figura 2).

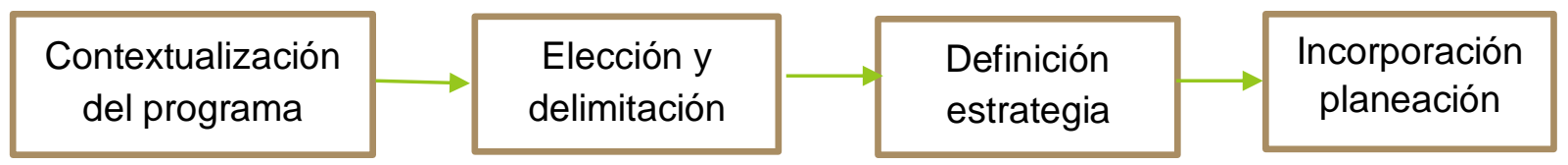

Figura 2. Fases de la metodología Lumbi-Castaño.

- Fase de Contextualización.En la fase de contextualización, se propone:

Identificar los elementos del programa de asignatura, que permita la integración del espíritu emprendedor, de acuerdo al siguiente orden :

1- Descripción de componente formativo. En este punto se pretende verificar que el componente espíritu emprendedor se encuentre oficializado por la institución en la asignatura y definido el alcance o finalidad esperada con relación a las temáticas que se abordarán.

2- Características que se pretenden fomentar en los estudiantes. La descripción del componente debe indicar que características se requieren potencializar a través de la asignatura.

La combinación de estos elementos permitirá la selección de las estrategias de aprendizaje a utilizar durante el desarrollo de la asignatura.

- Fase de elección y delimitación

Una vez que se han definido las características del espíritu emprendedor a desarrollar, se procederá a seleccionar en cual de las unidades temáticas se incorporarán las estrategias de aprendizaje.

1- Selecciona una unidad temática del programa de asignatura, identifica los objetivos particulares, especificamente el objetivo actitudinal y los contenidos a desarrollar.

2- Selecciona en el plan calendario de la asignatura, las formas organizativas de enseñanza (FOE), y las actividades de aprendizaje correspondientes a esa unidad temática. Se debe considerar que las formas de organización, pueden contener la definición de más de una actividad de aprendizaje.

3- Delimita el subtema que puede propiciar el desarrollo de las carácterísticas emprendedoras planteadas en el componente formativo y en el objetivo actitudinal.

Fase de definición de estrategias de aprendizaje

El Higo Revista Científica / Volumen 10. No. 01, pp. 11-37 / Junio 2020 
Cuando se habla de estrategias de aprendizaje, los autores implicitamente se refieren a formas de trabajar mentalmente que se supone o han probado que mejoran el rendimiento y evitan el fracaso. Son formas de aprender más y mejor con el mismo esfuerzo. Así mismo se describen como un conjunto de procesos o actividades que el aprendiz pone en funcionamiento con el propósito de facilitar la adquisición, el almacaneminto y/o la utilización de la información (González Pienda y Nuñez, 2006).

La competencia de aprender a aprender ha sido definida por la Comisión y el parlamento europeo como una competencia clave para el aprendizaje permanente y la define como vinculada a la capacidad de emprender y organizar un aprendizaje, ya sea individualmente o en grupos, según las necesidades propias del individuo, ser conscientes de los métodos y determinar las oportunidades disponibles.

Tomando en cuenta lo antes referido, algunas estrategias de aprendizaje que se pueden emplear para desarrollar las competencias emprendedoras, se muestran en la tabla 1.

Tabla 1. Estrategias de aprendizaje y competencias emprendedoras

\begin{tabular}{|c|c|c|}
\hline No. & Estrategia de aprendizaje & Competencias emprededoras a desarrollar \\
\hline 1 & $\begin{array}{l}\text { Estudio de casos: consiste } \\
\text { en analizar, gestionar, tomar } \\
\text { decisiones y buscar } \\
\text { soluciones eficaces a } \\
\text { problemáticas reales de } \\
\text { actualidad o simuladas (De } \\
\text { Miguel, 2006; Jiménez, } \\
2015 ; \text { Labrador y Andreu, } \\
\text { 2008; Fernández, 2006; } \\
\text { Zabala y Arnau, 2014) y que } \\
\text { está abierta a distintas } \\
\text { interpretaciones (Coloma, } \\
\text { Jiménez y Sáez, 2007). El } \\
\text { objetivo es acercar al } \\
\text { alumno a su futura profesión } \\
\text { (Labrador y Andreu, 2008). }\end{array}$ & $\begin{array}{ll}\text { - } & \text { Pensamiento crítico (Jiménez, 2015; Zabala y } \\
& \text { Arnau, 2014) } \\
\text { - } & \text { Comunicación oral y escrita (Jiménez, 2015; De } \\
& \text { Miguel, 2006) } \\
\text { - } & \text { Resolución de problemas (Jiménez, 2015; Zabala y } \\
& \text { Arnau, 2014) } \\
\text { - } & \text { Trabajo en equipo (Jiménez, 2015; Zabala y Arnau, } \\
& \text { 2014) } \\
\text { - } & \text { Planificación (Coloma, Jiménez y Sáez, 2007; De } \\
& \text { Miguel, 2006) } \\
\text { - } & \text { Toma de decisiones (Coloma, Jiménez y Sáez, } \\
& \text { 2007; Zabala y Arnau, 2014) } \\
\text { - } & \text { Visión de futuro (Coloma, Jiménez y Sáez, 2007) } \\
\text { - } & \text { Motivación para el logro (Coloma, Jiménez y Sáez, } \\
& \text { 2007) } \\
\text { - } & \text { Iniciativa (De Miguel, 2006) } \\
\text { - } & \text { Responsabilidad (De Miguel, 2006) } \\
\text { - } & \text { Autonomía (De Miguel, 2006) } \\
\text { - } & \text { Comunicación interpersonal (Zabala y Arnau, 2014) }\end{array}$ \\
\hline No. & Estrategia de aprendizaje & Competencias emprededoras a desarrollar \\
\hline 2 & $\begin{array}{l}\text { Simulaciones y juegos: } \\
\text { permite acercar al alumno a } \\
\text { experiencias y desafíos } \\
\text { reales (Jiménez, 2015) }\end{array}$ & $\begin{array}{ll}\text { - } & \text { Creatividad (Jiménez, 2015) } \\
\text { - } & \text { Iniciativa (Jiménez, 2015) } \\
\text { - } & \text { Capacidades interpersonales (Fernández, 2006) - } \\
\text { - } & \text { Toma de decisiones (Zabala y Arnau, 2014) } \\
\text { - } & \text { Autonomía (Zabala y Arnau, 2014) }\end{array}$ \\
\hline
\end{tabular}

El Higo Revista Científica / Volumen 10. No. 01, pp. 11-37 / Junio 2020 


\begin{tabular}{|c|c|c|}
\hline 3 & $\begin{array}{l}\text { Aprendizaje basado en } \\
\text { proyectos: consiste en } \\
\text { diseñar, elaborar y realizar } \\
\text { un proyecto con el objetivo } \\
\text { de resolver problemas } \\
\text { auténticos o abordar una } \\
\text { tarea (De Miguel, 2006; } \\
\text { Jiménez, 2015) }\end{array}$ & $\begin{array}{ll}\text { - } & \text { Pensamiento crítico (Jiménez, 2015) } \\
\text { - } & \text { Trabajo en equipo (Jiménez, 2015; De Miguel, } \\
\text { 2006) } & \\
\text { - } & \text { Comunicación oral y escrita (Jiménez, 2015; De } \\
& \text { Miguel, 2006) } \\
\text { - } & \text { Pensamiento creativo (Fernández, 2006) } \\
\text { - } & \text { Toma de decisiones (De Miguel, 2006) } \\
\text { - } & \text { Iniciativa (De Miguel, 2006) } \\
\text { - } & \text { Planificación (De Miguel, 2006) } \\
\text { - } & \text { Responsabilidad (De Miguel, 2006) }\end{array}$ \\
\hline 4 & $\begin{array}{l}\text { Aprendizaje cooperativo: } \\
\text { Trabajo en grupos reducidos } \\
\text { (Jiménez, 2015) para lograr } \\
\text { metas comunes (Labrador y } \\
\text { Andreu, 2008). No se debe } \\
\text { confundir el aprendizaje } \\
\text { cooperativo con el trabajo } \\
\text { en grupo. Este último suele } \\
\text { tender a ser competitivo } \\
\text { (Coloma, Jiménez y Sáez, } \\
\text { 2007). El aprendizaje } \\
\text { cooperativo, por el contrario, } \\
\text { es en colaboración entre } \\
\text { iguales (Coloma, Jiménez y } \\
\text { Sáez, 2007; Labrador y } \\
\text { Andreu, 2008). Esto es, se } \\
\text { da una estrategia de } \\
\text { corresponsabilidad para } \\
\text { lograr metas grupales (De } \\
\text { Miguel, 2006) dado que uno } \\
\text { alcanza sus objetivos si, y } \\
\text { sólo si, los demás consiguen } \\
\text { los suyos. Gracias a la zona } \\
\text { de desarrollo próximo el } \\
\text { sujeto puede adquirir } \\
\text { nuevos contenidos, resolver } \\
\text { problemas y realizar tareas } \\
\text { (De Miguel, 2006) }\end{array}$ & 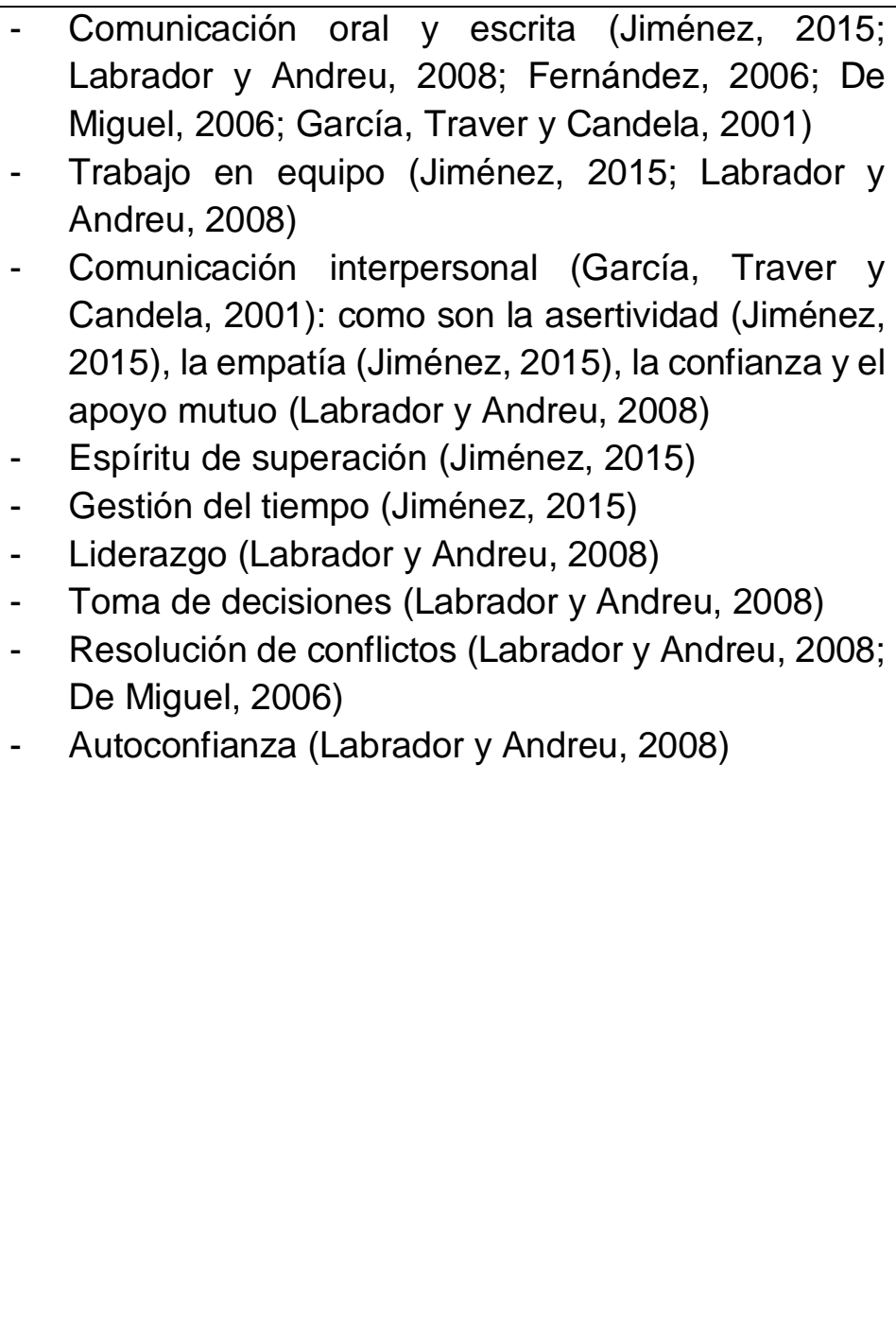 \\
\hline No. & Estrategia de aprendizaje & Competencias emprededoras a desarrollar \\
\hline 5 & 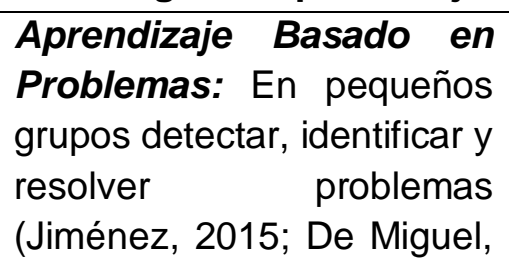 & $\begin{array}{l}\text { - Pensamiento crítico (Jiménez, 2015; Labrador y } \\
\text { Andreu, 2008; Zabala y Arnau, 2014) } \\
\text { Trabajo en equipo (Jiménez, 2015; De Miguel, } \\
\text { 2006; Zabala y Arnau, 2014) }\end{array}$ \\
\hline
\end{tabular}




\begin{tabular}{|l|l|l|l|}
\hline 2006; Fernández, 2006; & - & Resolución de problemas (Jiménez, 2015; Moust, \\
Labrador y Andreu, 2008; & Bouhuijs y Schmidt, 2007; De Miguel, 2006; Zabala \\
Zabala y Arnau, 2014) & yrnau, 2014) \\
poniendo en práctica los \\
conocimientos previos \\
(Moust, Bouhuijs y Schmidt, \\
2007).
\end{tabular}

Una vez definidas las características o competencias emprendedoras a desarrollar durante el desarrollo de la unidad temática, se debe analizar que tipo de estrategias de aprendizaje a utilizar en dependencia del contexto de la asignatura.

Fase de incorporación en la planeación didáctica

Una vez tomada la decisión del tipo de estrategia a utilizar, se debe incorporar en el plan calendario de la asignatura la estrategia de aprendizaje, especificando:

- Las características emprendedoras que se desean potenciar

- Los recursos didacticos que se utilizarán

- Criterios de evaluación que se considerarán para determinar el nivel de adquisición y/o fortalecimiento de las carácterísticas emprendedoras.

Cabe indicar que en la planeación didactica, se pueden incluir tantas estrategias de aprendizajes orientadas a desarrollar el espíritu emprendedor, como el docente lo estime y lo dicten los objetivos actitudinales definidos en el programa de asignatura, de esta forma, una vez que se concluya la ejecución de la asignatura, se podrá generar un informe de resultados en el que se indique de que forma la asignatura ha contribuido al desarrollo de esta competencia en los estudiantes.

Con el objetivo de comprobar la efectividad de la metodología, se incorporaron estrategias de aprendizaje que permiten el desarrollo del espíritu emprendedor en las planeaciones didacticas de dos asignaturas tomadas como pruebas pilotos, una con 27 estudiantes de la carrera de ingeniería eléctrica en la asignatura de química general, y la otra con 30 estudiantes de cuarto año de la carrera de ingeniería de sistemas, en la asignatura sistemas operativos.

La aplicación de la metodología, se realizó de la siguiente manera en el programa de la asignatura "Química General": 


\section{Fase de contextualización:}

Elementos identificados:

- Componente formativo: Espíritu emprendedor: A través de los trabajos extraclases, trabajos de investigación e informes de prácticas de laboratorio, el estudiante desarrollo las características del emprendedor; Liderazgo, proactividad, perseverancia, motivación, trabajo en equipo, y aprendizaje premanente.

\section{Fase de elección y delimitación}

Unidad temática: Conceptos básicos y ley periódica

Subtema: Celdas electroquímicas

Objetivo actitudinal: Valorar criticamente el aporte de la química en el desarrollo de la sociedad actual, considerando tanto sus logros como su impacto en el medio ambiente y en la salud

\section{Fase de definición de la estrategia de aprendizaje}

Estrategia de aprendizaje: Aprendizaje cooperativo Actividad de aprendizaje: Trabajo grupal- Visualización creativa: elaboración de brochure acerca de la implementación en la industria de metales, no metales y metaloides y elaboración de maqueta de celdas electroquímicas con materiales reciclados.

\section{Fase de incorporación}

Características a desarrollar: Liderazgo, proactividad, perseverancia, motivación, trabajo en equipo, y aprendizaje premanente

Recursos didacticos: T.P (Tabla periódica), Material de estudio de la primera unidad, Video: Tabla Periódica: Metales, no metales y metaloides. https://www.youtube.com/watch?v=WBeaTwmssxc. Tutorial: Como hacer el diseño de un brochure. http://espana.brochuredesianteam.com/Diseno-Brochure-1500.html. Guía de trabaio

Criterios de evaluación: Evidencia de aprendizaje: Brochure Implementación en la industria de metales, no metales, metaloides Ejemplos. Coevaluación, Aspectos a evaluar: Expresión oral y aportes pertinentes a la clase. Participación activa, dinámica, respetuosa de los diferentes puntos de vista. Presentación y dominio del tema. Características emprendedoras: Innovación y Creatividad, Fijación de objetivos, Toma de decisiones, Iniciativa y proactividad, Pensamiento crítico, Contextualización. 
La actividad de aprendizaje se ejecutó con la participación activa de los estudiantes, tal como se muestra en las siguientes imágenes.
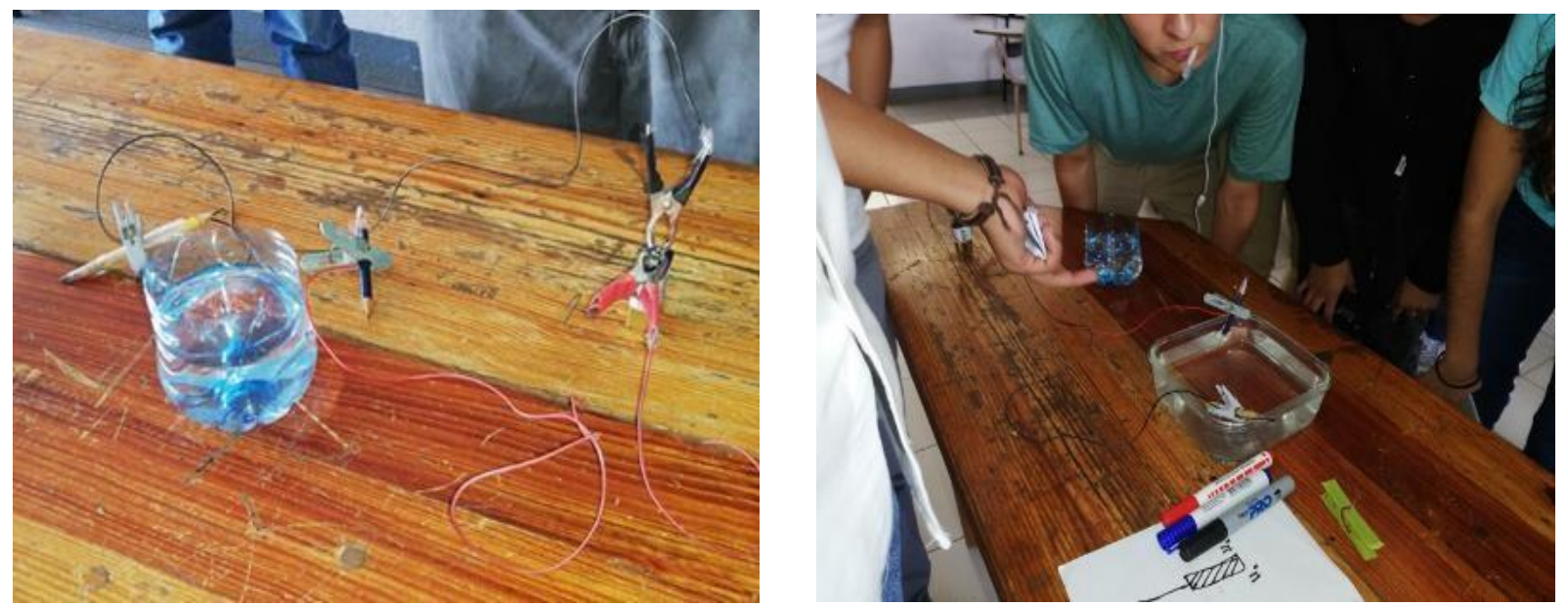

Figura 3. Proceso para elaboración de maquetas de una celda electroquímica con materiales reciclados.

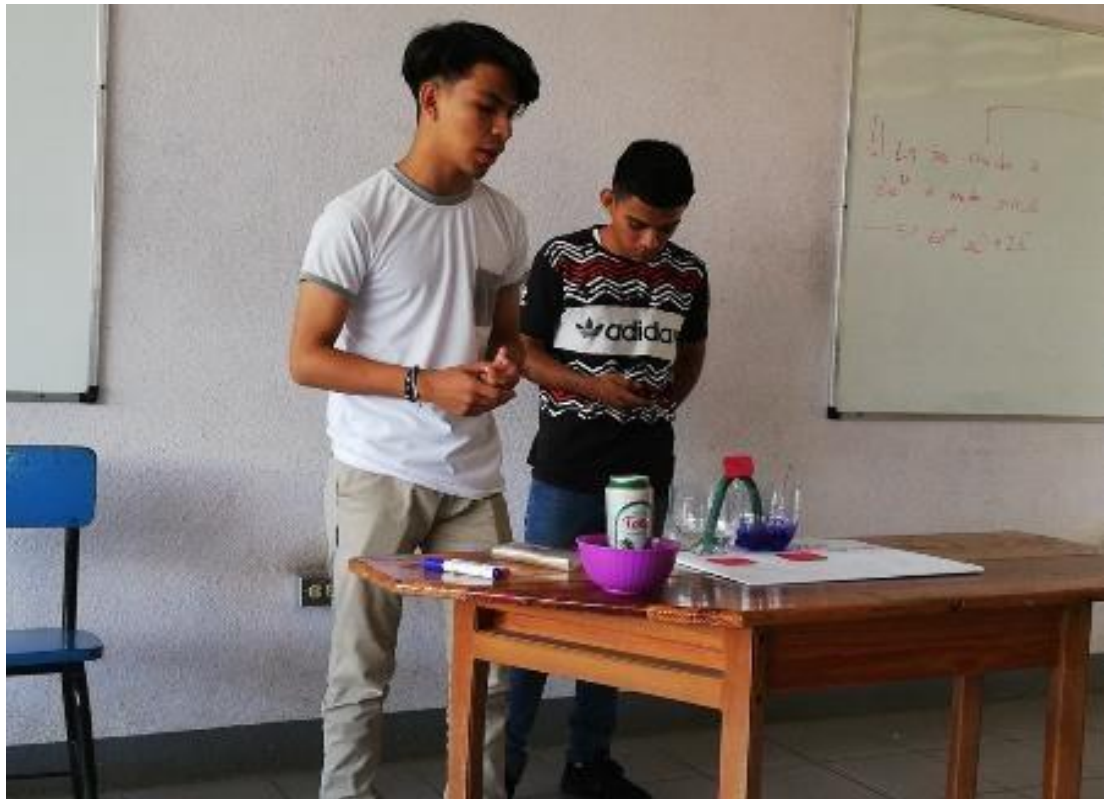

Figura 4. Exposición de estudiantes explicando el funcionamiento de las celdas electroquímicas elaboradas con material reciclado. 
En lo referido a los 27 estudiantes de la carrera de ingeniería eléctrica a través de la asignatura de química general, se realizó valoración de los estudiantes del trabajo realizado con esta metodología por medio de dinámicas de socialización y entrevistas grupales que permitían expresar los principales aprendizajes alcanzados y los resultados fueron:

$\checkmark \quad$ Fortalecimiento de las características emprendedoras a nivel personal.

$\checkmark \quad$ Desarrollo del trabajo colaborativo.

$\checkmark \quad$ Fortalecimiento de habilidades de comunicación.

$\checkmark \quad$ Desarrollo de relaciones interpersonales, les permitió conocerse entre los estudiantes y poder socializar para el desarrollo exitoso de las estrategias de aprendizaje. $\checkmark \quad$ Fomento de las competencias del programa Aprender, Emprender, Prosperar, específicamente: autocontrol, autoconfianza, razonamiento lógico, comunicación.

$\checkmark \quad$ Desarrollo de la creatividad, innovación, a través de la puesta en práctica de cada una de las estrategias de aprendizaje.

$\checkmark \quad$ Motivación por el aprendizaje de la química a través del aprender haciendo.

Todos los estudiantes expresaron que además de los logros de aprendizaje antes expuestos, se sentían motivados por aprender, investigar y sentían que practicaban haciendo ellos mismos sus evidencias porque las lograban relacionar con el contexto,

La aplicación de la metodología en la asignatura: Sistemas Operativos, durante el segundo semestre del año 2019, en la planificación didáctica se incorporaron diversas estrategias de aprendizaje, con la finalidad de desarrollar y potencializar el espíritu emprendedor.

Las estrategias de aprendizaje desarrolladas fueron las siguientes:

- $\quad$ Dramatizaciones/sociodramas (Aprendizaje colaborativo)

- $\quad$ Organización de información - Mapa conceptual (Aprendizaje colaborativo)

- $\quad$ Organización de información - Elaboración de Resumen (Aprendizaje colaborativo)

- $\quad$ Tarea de curso (Aprendizaje interactivo)

- $\quad$ Foros virtuales (Aprendizaje colaborativo)

- $\quad$ Solución de ejercicios (Autoaprendizaje)

Para todas las estrategias de aprendizaje, se desarrollaron los respectivos guiones de trabajo que incluyeron:

- $\quad$ Descripción de la estrategia (procedimientos a seguir)

- Objetivos

- $\quad$ Subtemas a desarrollar

- Recursos disponibles

- $\quad$ Criterios de evaluación

En las siguientes imágenes se muestran a los estudiantes trabajando en el desarrollo de las estrategias de aprendizaje: 

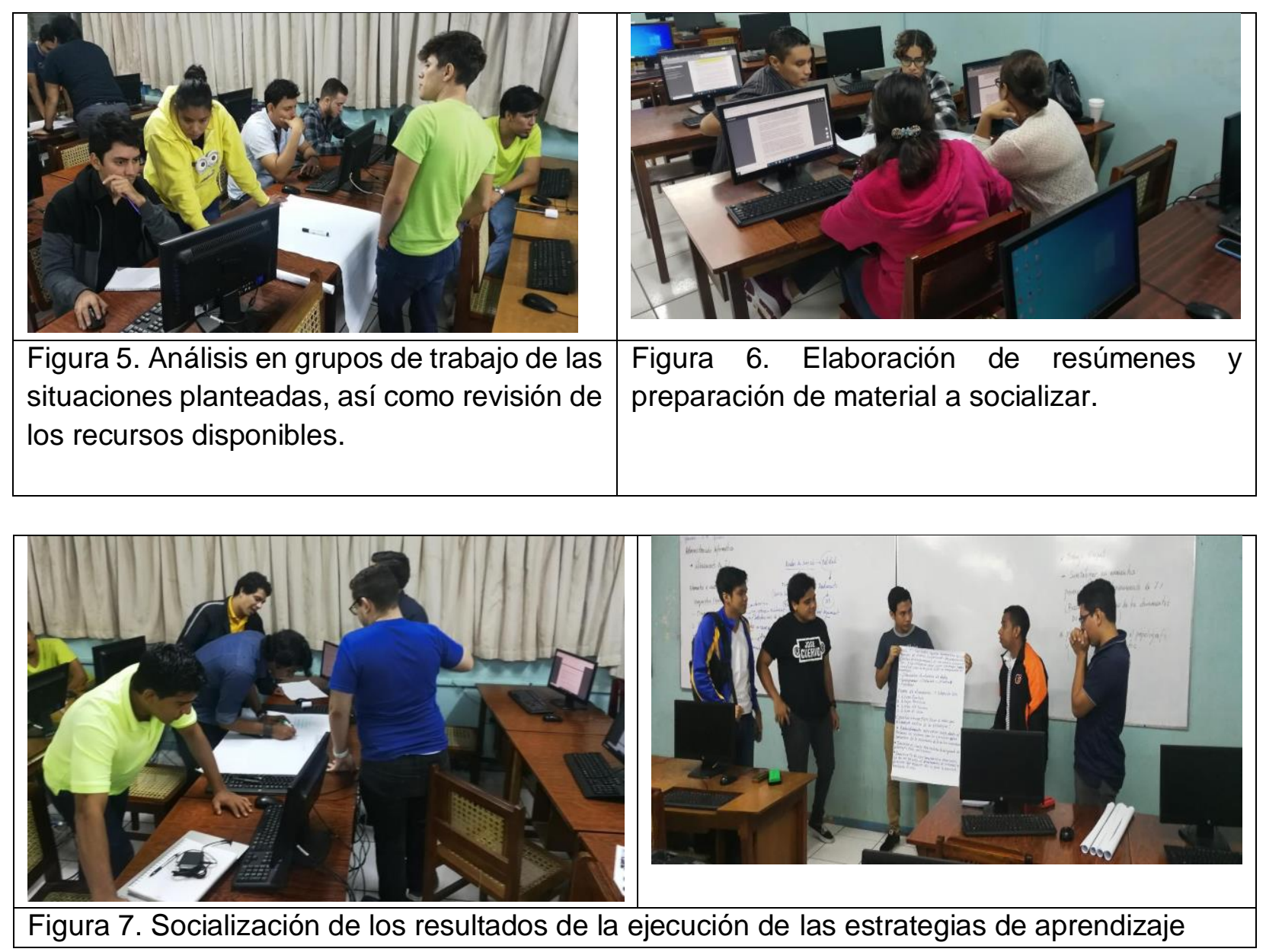

En cuanto a los resultados de los 30 estudiantes de cuarto año de la carrera de ingeniería de sistemas se determina el impacto obtenido en los estudiantes, mediante la aplicación de una encuesta rápida, los resultados se muestran a continuación:

El 85\% de los estudiantes del grupo (25), participaron en todas las estrategias de aprendizaje, tal como se muestra en la siguiente figura, siendo las dramatizaciones/sociodramas, la de mayor participación con un $93.3 \%$ (28 estudiantes). 
Olga del S. Rodríguez; Reynaldo A. Castaño U.

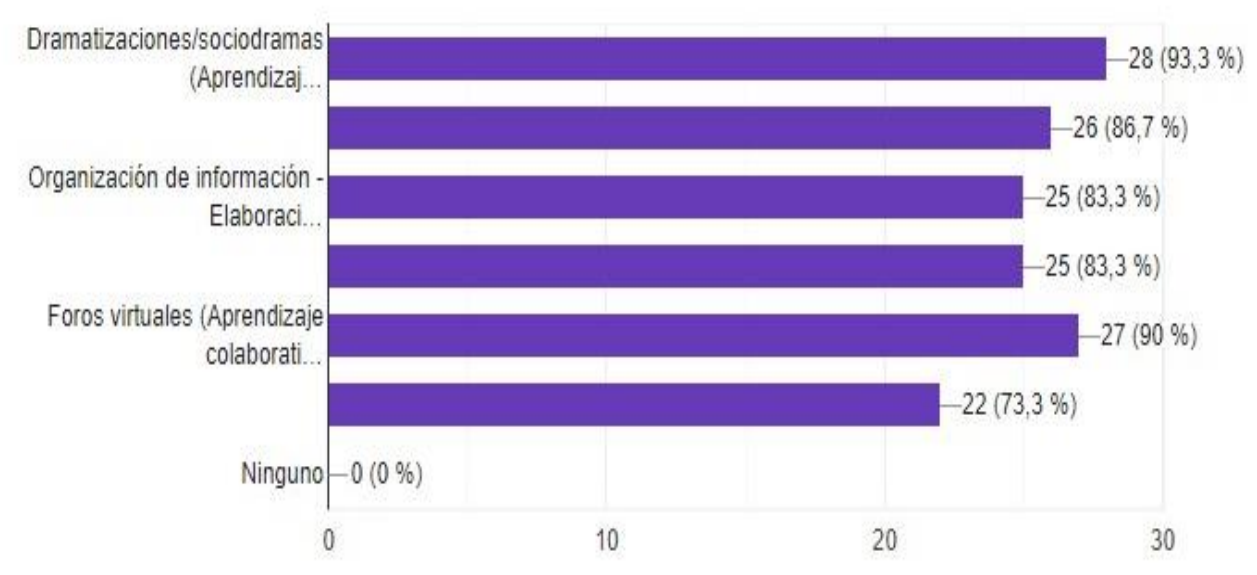

Figura 8. Participación de los estudiantes en las diferentes estrategias de aprendizaje desarrolladas.

El 93,4\% de los estudiantes (28), expresan que la utilización de estrategias de aprendizaje, favorece la construcción y consolidación de los conocimientos, tal como se muestra en la figura 9.

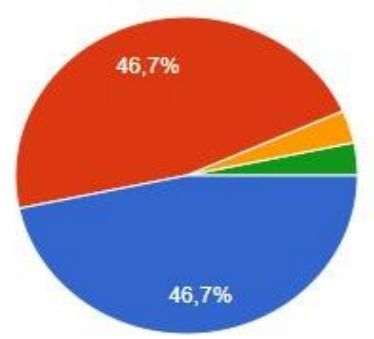

Totalmente de acuerdo

De acuerdo

Ni de acuerdo ni en desacuerdo

En desacuerdo

Totalmente en desacuerdo

Figura 9. Estrategias de aprendizaje utilizadas por el docente, favorece construcción y consolidación

El 90\% (27) de los estudiantes, coinciden en que las estrategias de aprendizaje favorecen el desarrollo de actitudes y valores, tal como se muestra en la figura 10.

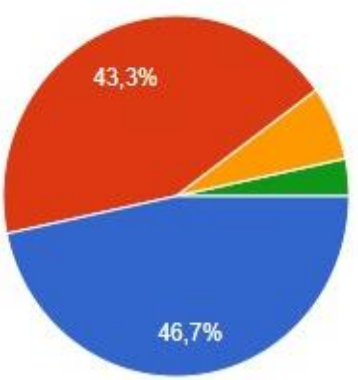

Totalmente de acuerdo

De acuerdo

Ni de acuerdo ni en desacuerdo

En desacuerdo

Totalmente en desacuerdo

Figura 10. El tipo de estrategias de aprendizaje implementadas permiten el desarrollo de actitudes y valores.

El Higo Revista Científica / Volumen 10. No. 01, pp. 11-37/ Junio 2020 
Según la apreciación de los estudiantes, a través de las estrategias de aprendizaje, se desarrollan varias actitudes y características, siendo las características más desarrollada; el trabajo en equipo (con un $93,3 \%$ ), en segundo lugar, la creatividad e innovación (con un $70 \%$ ) y la toma de decisiones (70\%) y en tercer lugar el desarrollo del pensamiento crítico (56.7\%), en menor cuantía: búsqueda de resultados, iniciativa, liderazgo, creatividad, pensamiento crítico entre otras mostradas en la siguiente figurar.

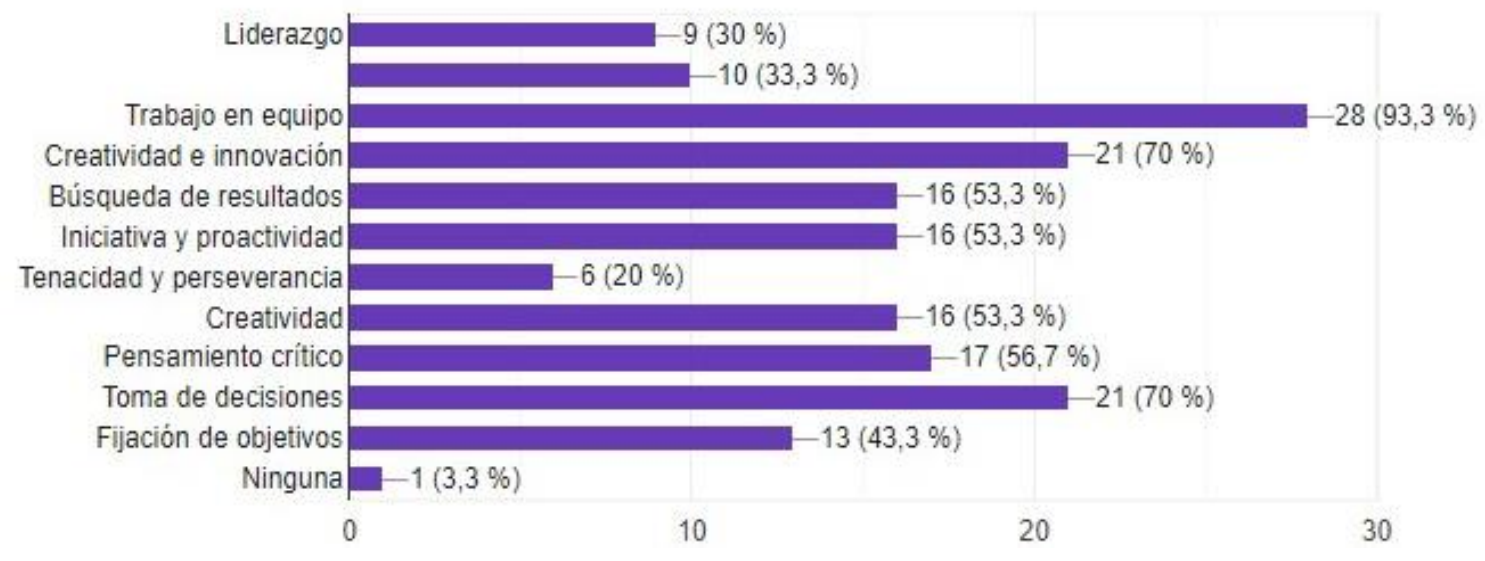

Figura 11. Indique que actitud o características logró desarrollar durante la ejecución de las estrategias de aprendizaje.

El $93.4 \%$ (28) de los estudiantes, expresan estar de acuerdo con el empleo de estrategias de aprendizaje en el desarrollo de su proceso de formación, a través de las diferentes asignaturas que conforman el plan de estudio, tal y como se visualiza en la figura 13.

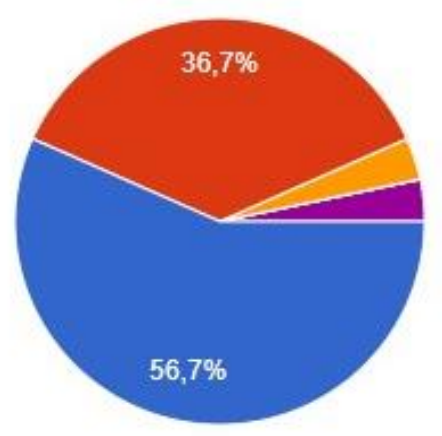

Totalmente de acuerdo

De acuerdo

Ni de acuerdo ni en desacuerdo

En desacuerdo

Totalmente en desacuerdo

Figura 12. Valoracion en la incorporación de estrategias de aprendizaje en el proceso de formacion.

Una vez validada la metodología Lumbí-Castaño, mediante la puesta en práctica en las dos asignaturas citadas anteriormente, se procedió a diseñar un taller orientado al trabajo con los El Higo Revista Científica / Volumen 10. No. 01, pp. 11-37/ Junio 2020 
docentes de la Universidad con la finalidad de fortalecer el quehacer docente incorporando una metodología que permita la inserción del espíritu emprendedor en la planificación didáctica.

Bajo esa premias y a través del diseño de esta metodología holística y de carácter practcio se logro capacitar por primera vez durante este taller a 43 docentes desde su planificación didáctica en las facultades de Ciencias y Sistemas, Tecnología de la construcción y arquitectura, tal como se muestra en la tabla 2.

Tabla 2. Cantidad de docentes capacitados por facultades.

\begin{tabular}{|l|c|}
\hline Facultad & Cantidad de docentes \\
\hline Ciencias y Sistemas & 11 \\
\hline Arquitectura & 17 \\
\hline Tecnología de la construcción & 12 \\
\hline Coordinador de extensión FIQ & 1 \\
\hline Coordinador de FTC & 1 \\
\hline Coordinador de FCYS & 1 \\
\hline
\end{tabular}

Para el fortalecimiento de la formación académica en Emprendimiento es necesario que el docente ponga en práctica, metodologías, enfoques y estrategias didácticas que medien exitosamente en la formación de estudiantes proactivos, que permitan abordar en los procesos de aprendizaje y enseñanza; enfoques diferentes a los tradicionales.

Con la realización de este taller se confirma que, para fomentar el espíritu emprendedor desde el quehacer didáctico, debe contarse con el aprovechamiento de ambientes de aprendizaje idóneos, utilizando enfoques metodológicos interactivos.

Se requiere sensibilizar y concientizar a los docentes en la temática del emprendimiento, pero desde una perspectiva holística, enfatizando en el desarrollo de las características del SER emprendedor.

Se deben diseñar estrategias de aprendizaje que permitan el fomento del aprendizaje activo, mejorando la experiencia y brindando oportunidades para el diálogo reflexivo.

Se recomienda fortalecer las funciones de afectividad positiva en el docente, para que ponga en práctica la evaluación de los estudiantes haciendo énfasis en el reconocimiento a los avances en su proceso formativo, elogiándolo, estimulándolo, expresando aprecio y confianza en sus estudiantes.

Se requiere preparar al docente mediante procesos de formación, capacitación y actualización en los temas de emprendimiento, pero no solamente desde la perspectiva de emprendimiento empresarial, sino también para desarrollar las características emprendedoras en los estudiantes. 
Se debe propiciar la incorporación del docente en planes y proyectos de emprendimiento que le permitan el desarrollo de experiencias empresariales, así como el fortalecimiento de sus características y competencias.

Como resultado de esta investigación, se obtuvieron los siguientes productos:

$\checkmark 43$ Docentes capacitados en la utilización de estrategias didácticas dinámicas, motivadoras y prácticas que promueven la inserción de las características emprendedoras desde su planificación didáctica.

$\checkmark$ Puesta en práctica de trabajo colaborativo de dos docentes creadores de la metodología.

$\checkmark$ Fomento del espíritu emprendedor en la planificación didáctica.

$\checkmark 57$ estudiantes motivados a desarrollar sus características emprendedoras personales a través del desarrollo de las estrategias didácticas que evalúan características emprendedoras.

$\checkmark$ Metodología Lumbí-Castaño, puesta en práctica y validada su eficacia en el desarrollo del espíritu emprendedor de los estudiantes.

\section{CONCLUSIONES}

Con el desarrollo de esta investigación, se logró determinar que aún no se cuenta con una metodología única que facilite la inserción del espíritu emprendedor en los estudiantes durante el desarrollo de las asignaturas, por lo que los docentes carecen hasta el momento de una guía metodológica que oriente de forma eficiente las fases a seguir durante la planeación didáctica.

Las características o competencias emprendedoras en los estudiantes, se pueden desarrollar desde cualquier asignatura del pensum académico, mediante la definición de estrategias de aprendizajes dinámicas centradas en el estudiante (aprendizaje activo).

La metodología propuesta, denominada Lumbí-Castaño, para la incorporación de estrategias de aprendizaje en la planeación didáctica de cualquier tipo de asignatura, y con la finalidad de propiciar el desarrollo de las características emprendedoras en los estudiantes, tales como: originalidad, trabajo en equipo, creatividad, comunicación, entre otras, ha demostrado ser eficaz en la ejecución de las asignaturas, facilitando la incorporación activa de los estudiantes en las actividades académicas, obteniendo las siguientes percepciones:

Más del 90\% de los estudiantes:

$\checkmark$ Encontró efectiva la utilización de estrategias de aprendizaje para fortalecer los conocimientos

$\checkmark$ Expresó, que a través del desarrollo de las estrategias adquirieron y/o desarrollaron algún tipo de característica del espíritu emprendedor, siendo las sobresalientes: trabajo en equipo y toma de decisiones.

El Higo Revista Científica / Volumen 10. No. 01, pp. 11-37/ Junio 2020 
Olga del S. Rodríguez; Reynaldo A. Castaño U.

$\checkmark$ Está de acuerdo con la incorporación de estrategias de aprendizaje en el desarrollo de las asignaturas del plan de estudio.

\section{REFERENCIAS}

Alcaraz, R. (2015). El emprendedor de éxito. Quinta edición. México: Mc Graw Hill / Interamericana editores S.A. de C.V

Álvarez, P.R. (2016). Competencias genéricas en la enseñanza universitaria. De la tutoría formativa a la integración curricular. Málaga: Ediciones Aljibe.

Arasti, Z., Kiani, M. y Imanipour, N. (2012). A Study of Teaching Methods in Entrepreneurship Education for Graduate Students. Higher Education Studies, 2(1), 2-10.

Arruti, A. (2016). El desarrollo del perfil del teacherpreneur o profesor-emprendedor en el currículum del Grado de Educación Primaria: ¿un concepto de moda o una realidad? Contextos educativos, 19, 177-194.

Aquije, M. (2012). Tú eres un emprendedor: Editorial San Marcos. Lima Perú

Coloma, A. M., Jiménez, M. Á. y Sáez, A. M. (2007). Metodologías para desarrollar competencias y atender a la diversidad. Guía para el cambio metodológico y ejemplos desde infantil hasta la universidad. Madrid: PPC Editorial. Comisión Europea (2008). Entrepreneurship in Higher Education, especially in non-business studies. Comisión Europea (2016a).

Dacin, T.; Dacin, P.A. Y Tracey, P. (2011) Social entrepreneurship: a critique and future directions. Organization Science, vol. 22, nำ5, pp. 1203-1213.

De la Fuente, J., Vera, M.M. y Cardelle-Elawar, M. (2012). Aportaciones de la Psicología de la Innovación y del Emprendimiento a la Educación en la Sociedad del Conocimiento. Electronic Journal of Research in Educational Psychology, 10(3), 941-966.

De Miguel, M. (Coord.) (2005). Modalidades de Enseñanza centradas en el desarrollo de Competencias: orientaciones para promover el cambio metodológico en el Espacio Europeo de Educación Superior. Proyecto EA2005-0118.

De Miguel, M. (Coord.) (2006). Metodologías de enseñanza y aprendizaje para el desarrollo de competencias. Orientaciones para el profesorado universitario ante el Espacio Europeo de Educación Superior. Madrid: Alianza Editorial.

Fayolle, A. y Gailly, B. (2008) From craft to science. Teaching models and learning processes in entrepreneurship education. Journal of European Industrial Training, vol. 32, no 7, pp. 569-593.

El Higo Revista Científica / Volumen 10. No. 01, pp. 11-37/ Junio 2020 
Fernández, A. (2006). Metodologías activas para la formación de competencias. Educatio siglo XXI, 24, 35-56.

Ferrer-Cerveró, V., Cabrera-Santacana, O. E., Alegre-Beneria, R. M., Montané-Lopez, A., Sánchez-Valverde-Visus, C., y Alaiz-ChuecA, E. (2014) El perfil del emprendedor social del estudiantado de los Grados de Educación Social, Pedagogía y Trabajo Social en la Universidad de Barcelona. [En línea] REIRE, Revista d'Innovació i Recerca en Educació, vol. 7, n 1. Disponible en: http://www.ub.edu/ice/reire.html. ISSN: 2013-2255.

García, R., Traver, J.A. y Candela, I. (2001). Aprendizaje cooperativo. Fundamentos, características y técnicas. Madrid: Editorial CCS.

González, J. y Wagenaar, R. (Eds.). (2003). Tuning Educational Structures in Europe. Informe Final. Fase Uno. Deusto: Universidad de Deusto.

Jiménez, G., Elías, R. y Silva, C. (2014). Innovación docente y su aplicación al EEES: Emprendimiento, TIC y Universidad. Historia y Comunicación Social, 19, 187-196.

Jiménez, R. (Coord.). (2015). Educación emprendedora. Programa TALOS para el desarrollo de la iniciativa emprendedora en Ciencias de la Educación. Barcelona: Editorial Octaedro.

Jones, C.; Matlay, H. Y MaritZ, A. (2012) Enterprise education: for all, or just some? Education +Training, vol. 54, no 8/9, pp. 813-824.

Korainen, m. y Ruohotie, P. (2001. In the Pursuit of Conative Constructs into Entrepreneurship Education. Journal of Entrepreneurship Education, vol. 3, pp. 9-22.

Korsgaard, S. (2011) Opportunity formation in social entrepreneurship. Journal of Enterprising Communities: People and places in the global economy, vol. 5, no 4, pp. 265- 285.

Labrador, M. J. y Andreu, M. Á. (2008). Metodologías activas. Grupo de Innovación en metodologías activas (GIMA). Valencia: Editorial UPV.

Merino, M. and Vargas, D. (2011), "Evaluación comparativa del potencial emprendedor de Latinoamérica: una perspectiva multinivel", Academia Revista Latinoamericana de Administración, Vol. 46 No. 46, pp. 38-54.

Miller, T.L.; Wesley II, C.L. y Williams, D.E. (2012) Educating the minds of caring hearts: comparing the views of practitioners and educators on the importance of social entrepreneurship competencies. Academy of Management Learning \& Education, vol. 11, № 3, pp. 349-370. 
Moust, J., Bouhuijs, P. y Schmidt, H. (2007). El aprendizaje basado en problemas: guía del estudiante. Cuenca: Ediciones de la Universidad de Castilla-La Mancha.

.Osorio, F.F. y Pereira, F. (2011) Hacia un modelo de educación para el emprendimiento: una mirada desde la teoría social cognitiva. Cuadernos de Administración, vol. 24, no 43, pp. 13-33.

Pache, A.C. y Chowdhury, I. (2012) Social entrepreneurs as institutionally embedded entrepreneurs: Toward a new model of social entrepreneurship education. Academy of Management Learning \& Education, vol. 11, nํㅜ 3, pp. 494-510.

Pfeilstetter, R. (2011) El emprendedor. Una reflexión crítica sobre usos y significados actuales de un concepto. Gazeta de Antropología, 27 (1). Disponible en: http://hdl.handle.net/10481/15684. ISSN: 0214-7564.

Raposo, M. Y Do Paço, A. (2011) Entrepreneurship education: Relationship between education and entrepreneurial activity. Psicothema, vol. 23, no 3, pp.453-457.

Ruskovaara, E. Y Pihkala, T. (2013) Teachers Implementing Entrepreneurship EducationClassroom Practices. Education + Training, vol. 55, no 2, pp. 204 -216.

Sánchez, J.C. y Gutiérrez, A. (2011) Entrepreneurship research in Spain: developments and distinctiveness. Psicothema 2011, vol. 23, no 3, pp.458-463.

Sánchez, J.C. (2013) The impact of an Entrepreneurship Education Program on entrepreneurial competencies and Intention. Journal of Small Business Management, vol. 51, nº 3, pp. 447-465.

Taatila, V.P. (2010) Learning entrepreneurship in higher education. Education + Training, vol. 52, no 1 , pp. 48-61.

Villanueva, S.D. (2013) Las competencias dentro del rol profesional: diferencias entre la Educación Superior (universitaria) y las demandas del mercado laboral. Revista Debate Universitario, [en línea] 1, (2m) pp. 44-65. Buenos Aires, CAEE-UAI. Disponible en: http://ppct.caicyt.gov.ar/index.php/debate-universitario/article/viewFile/1605/pdf. ISSN: 23141530.

Zabala, A. y Arnau, L. (2014). Métodos para la enseñanza de las competencias. Barcelona: Editorial Graó. 
Olga del S. Rodríguez; Reynaldo A. Castaño U.

\section{SEMBLANZA DE LOS AUTORES}

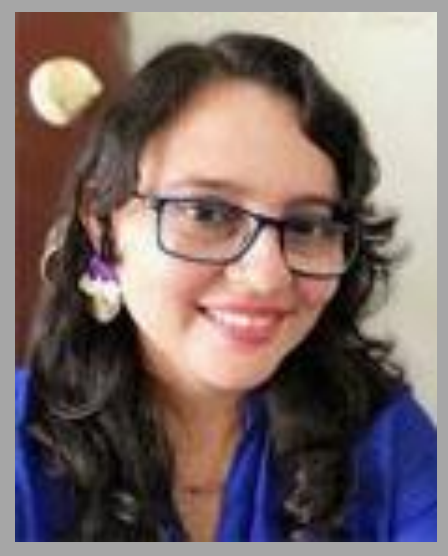

Olga del Socorro Rodríguez Lumbí: Ingeniera Química. Licenciada en Pedagogía. Máster en Didáctica para la enseñanza de Ingenieros y Arquitectos. Asesora Académica de la Dirección de Desarrollo Educativo de la Universidad Nacional de Ingeniería, UNI. Docente UNI de materias de Emprendimiento, Planificación Didáctica, Evaluación de los Aprendizajes, Química General, entre otras. Fue coordinadora de la Formación Emprendedora de la Facultad de Ingeniería Química de la UNI. Docente de la Carrera de Licenciatura en Educación con mención e innovación y emprendimiento de la Universidad Nacional Autónoma de Nicaragua, UNAN. Fue miembro de la Comisión Nacional de Emprendimiento del Programa Nacional Educativo Aprender, Emprender, Prosperar.

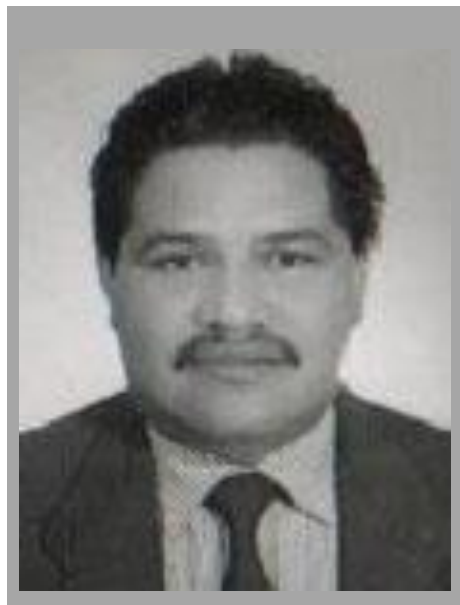

Reynaldo Antonio Castaño Umaña: Es graduado de Ingeniero en Automática, en la Universidad Central "Martha Abreu" de las Villas. Villa Clara. Cuba. Es máster en Redes de Computadoras, graduado en la Facultad de Electrotecnia y Computación de la Universidad Nacional de Ingeniería. Es profesor titular de la UNI, ha desempeñado tareas como Coordinador de Extensión de la Facultad de Ciencias y Sistemas, y como Coordinador de programas de Maestría. 